\title{
"Nous le verrons plus bas ", " voir ci-dessus », " je ne reviens pas ici »: retours sur les propriétés de la langue écrite
}

\author{
Julie Lefebvre \\ Université de Lorraine, CREM, EA 3476, Metz, F-57000, France.
}

\section{Introduction}

Soit les deux énoncés suivants ${ }^{1}$ :

Nous verrons que ce titre qui avait probablement séduit des chalands allait faire se lever des orages (...). [Lacouture J. (2000). Le témoignage est un combat - Une biographie de Germaine Tillion. Paris : Éditions du Seuil, p. 309]

Nous verrons plus loin que Germaine Tillion a nuancé cette affirmation. [Le témoignage est un combat, op.cit., p. 191]

Tous deux donnent à voir ce qu'on peut qualifier de « séquences », dans le sens où « nous verrons que » et «nous verrons plus loin que » se présentent comme des suites ordonnées de termes constituant un tout sous le rapport d'une activité donnée.

En tant qu'elles jouent un rôle dans l'économie communicationnelle du discours, ces séquences ont fait l'objet de travaux où elles sont analysées comme le lieu d'une activité métadiscursive — elles sont "à la fois discours et glose sur le discours dans lequel [elles sont] immergé[es]» (Borillo, 1985: 50) —, et apparaissent comme des «marqueurs de structuration, guidant le cours du raisonnement, facilitant le travail de présentation et par là même aidant à la compréhension » (ibid. : 56). Sans nous inscrire en faux contre ces analyses, c'est cependant d'un autre point de vue, énonciatif, que nous aborderons ces séquences. Empruntant une démarche similaire à celle d'Authier-Revuz (1995/2012) sur les «boucles réflexives du dire », nous considèrerons ces séquences comme autant d'espaces dans lesquels le locuteurscripteur laisse affleurer différents aspects de son rapport au dire en train de se faire.

Dans ce cadre, la différence qui existe entre les énoncés (1) et (2) cités ci-dessus, soit la présence, en (2) du syntagme adverbial «plus loin », est à prendre en compte. Dans une séquence du type de celle de l'exemple (1), pointant un futur de l'énonciation, le locuteur inscrit uniquement son dire dans une chronologie de la lecture-écriture. En revanche, dans une séquence du type de celle de l'exemple (2), c'est comme inscrit dans une temporalité et dans un espace particulier que le dire en train de se faire est représenté. L'activité métadiscursive à l'œuvre dans ces séquences se joue ainsi sur des arrière-plans distincts : le caractère linéaire du signifiant et l'inscription dans une temporalité qui lui est concomitante dans les séquences du type de celle de l'exemple (1) ; et les propriétés de la "langue écrite » (Vachek (1939), Catach (éd.) (1988), Anis (1988)) dans les séquences du type de celle de l'exemple (2).

Dans la présente communication, nous proposons de poser quelques jalons pour la description de cette deuxième sorte d'activité métadiscursive. Nous qualifions cette activité de "métascripturaire » en ceci qu'elle traite du dire en train de se faire en tant qu'il est écrit. Ce faisant, nous souhaitons montrer que, de la même manière que de nombreux travaux ont pu, ces dernières années notamment (autour, par exemple, du Groupe aixois de recherche en syntaxe), souligner les spécificités de l'oral, l'écrit a des propriétés spécifiques qui doivent être prises en compte dans l'analyse linguistique.

Notre étude sera menée à partir de l'observation de séquences métadiscursives comportant un syntagme adverbial (SAdv) autorisant un repérage spatial de type déictique, telles que « je ne reviens pas ici sur»" ou encore « nous verrons plus bas que ». Ces séquences sont extraites de différents textes relevant d'un genre discursif qu'on peut qualifier de scientifique ${ }^{2}$, leur fréquence élevée dans ce genre constituant en effet une base riche permettant de "saturer» une très grande partie de leurs réalisations, dont nous 
faisons l'hypothèse qu'on peut les retrouver dans d'autres genres discursifs (ainsi, par exemple, dans le champ littéraire).

La nature de l'activité réflexive à l'œuvre dans ces séquences sera principalement abordée à travers l'analyse des propriétés de l'emploi déictique de l'adverbe ici à l'écrit. L'examen de cette activité sera complété par une amorce de description des composés ci-dessus et ci-dessous, des groupes adverbiaux plus haut, plus bas et plus loin, et des adverbes latins «infra» et «supra». À travers quelques exemples, nous montrerons ensuite quelles perspectives ouvre, pour l'analyse des textes et des discours, l'attention portée aux "postures énonciatives » (Authier-Revuz,1995/2012) qui se dessinent dans ces séquences témoignant du rapport du locuteur - scripteur, en l'occurrence - à son «dire écrit». Enfin, en conclusion, nous évoquerons la place qu'occupent dans la sphère de l'activité métalangagière, relativement à d'autres énoncés métadiscursifs, ces séquences qui mettent en jeu les spécificités de la langue et de l'énonciation écrites.

\section{Ici dans son emploi déictique à l'écrit}

Les occurrences de l'adverbe ici qui nous intéressent sont toutes réalisées à l'écrit et permettent un repérage énonciatif de type déictique ${ }^{3}$. La description de ce qui est en jeu dans ce type de référence spatiale fournira un cadre pour la compréhension de l'activité métadiscursive à l'œuvre dans les séquences où elles s'inscrivent.

\subsection{Le « métascripturaire », secteur de l'activité métadiscursive}

Dans les exemples suivants, $i c i$ réfère à l'espace dans lequel se fait l'énonciation en cours :

Louis Perrin [...] nous donne ici son point de vue. [Cité dans Simonin, $1984: 196]$

(4)

Nous aurons l'occasion, dans les chapitres qui suivent, de retrouver, à propos des mécanismes de l'évolution humaine, plusieurs exemples de variabilité chimique des protéines. Nous n'en donnerons donc pas d'autres ici, nous contentant de rappeler les principales conclusions qui se dégagent de ces études [...]. [Langaney A. (1988). Les hommes - passé, présent, conditionnel. Paris : Armand Colin, p. 130-131]

Dans ces énoncés, il est fait référence à un espace qui, non géographique - il ne s'agit en effet pas du lieu physique dans lequel le scripteur prend place pour tenir son discours - , peut être caractérisé comme scripturaire, comme c'est le cas de l'espace du «journal» et de l'espace des lignes qui suivent l'occurrence de ici dans, respectivement, les exemples (3) et (4) ci-dessus. Une mise en perspective historique permettra de préciser ce qui est en jeu dans ces repérages spatiaux qu'autorise l'adverbe ici à l'écrit.

Le $c i$ « de référence textuelle » (Perret, 1988) apparaît massivement en moyen français dans les premiers textes de prose en langue vernaculaire. Ces derniers n'étant pas encore organisés en unités telles que les chapitres, la structuration du texte est assurée par des « énoncés internes annonçant la fin d'un épisode et le début d'un autre » (Perret, $1988: 108$ ), comme, par exemple, « cy commence l'ystoire [...] » (cité dans Perret, 1988 : 119). Ayant tendance à se substituer à l'adverbe or (maintenant) dans les énoncés qui ont pour fonction de structurer ces textes aux $\mathrm{XIV}^{\mathrm{e}}-\mathrm{XV}^{\mathrm{e}}$ siècles, le $c i$ « de référence textuelle » marque le passage d'un système de localisation temporelle à un système de localisation spatiale, passage résultant d'une « restructuration de l'espace énonciatif»:

la relation énonciative primaire entre le locuteur et son public qui avait été conçue, puis représentée comme immédiate, devient différée - conçue et représentée comme directe, elle devient médiatisée par l'écriture, le livre. Par une série de déplacements, l'espace énonciatif des premiers temps, espace indéfini où locuteur et public étaient en présence est devenu l'espace matériel du livre (Simonin, $1982: 181$ ). 
Ainsi, dans la lignée de ces $c i$ «de référence textuelle» observables en moyen français, ce que les occurrences de l'adverbe ici qui nous intéressent rendent saillant, du fait même de leur fonctionnement de type déictique, c'est la nature écrite de l'énonciation en train de se faire. En mettant au premier plan le fait que "l'énonciation, chaque fois unique, qui les contient ${ }^{4}$ se réalise dans un espace singulier constitué par le support et la ligne graphique qui s'y inscrit — seul espace commun, dans une temporalité du différé, au scripteur et aux lecteurs - elles « rappellent» le caractère écrit de l'énonciation en train de se faire. C'est à ce titre que nous proposons d'y voir une dimension « métascripturaire ».

\subsection{Référer au support de l'écrit, référer à la ligne écrite}

En moyen français aux $\mathrm{XIV}^{\mathrm{e}}-\mathrm{XV}^{\mathrm{e}}$ siècles, c'est une «sui-référentialité stricte » qui caractérise l'adverbe ci/ici dans son emploi relatif à la structuration du texte :

[le ci/ici de référence textuelle] apparaît dans des énoncés non négativables, au présent et au futur; il n'est pas paraphrasable par $c i / i c i+$ adverbe : c'est un $c i / i c i$ souvent performatif et toujours sui-référentiel au sens étroit: il ne désigne que la place occupée par ses graphèmes (Perret, 1988 : 106, c'est nous qui soulignons).

Cette sui-référentialité stricte du $\mathrm{ci} / \mathrm{ici}$ de structuration textuelle est cependant abandonnée dès la fin du $\mathrm{XV}^{\mathrm{e}}$ siècle. Une «sui-référentialité au sens large » la remplace alors, qui caractérisait déjà, un siècle auparavant, le $\mathrm{ci} / \mathrm{ici}$ ayant une fonction de «commentaire ou d'attestation » :

[le ci/ici de «commentaire ou d'attestation»] n'est presque jamais en début d'énoncé ; est paraphrasable par $c i+$ adverbe; il peut appartenir à un énoncé nié, à d'autres temps que le présent et le futur : c'est un $c i / i c i$ sui-référentiel au sens large : $i l$ désigne à la fois l'espace du texte où se produit l'occurrence et un espace antécédent, adjacent, suivant ou englobant (Perret, 1988 : 106, c'est nous qui soulignons).

Dans l'hypothèse de la permanence de ce caractère, on peut avancer que, en français contemporain et dans son emploi déictique à l'écrit, l'adverbe $i c i$ ne réfère pas « au lieu de l'occurrence » de $i c i$, mais « au lieu où est prononcée [son] occurrence » (Perret, $1988: 119)$.

Le lieu que désigne ici est de type différent selon que l' «extension » de l'espace auquel réfère ici relativement à la place qu'occupent ses graphèmes sur le support - se fait par « dilatation » autour de l'occurrence de ici qui est alors incluse dans l'espace en question, ou par "décalage » relativement à l'occurrence de ici qui n'est alors pas comprise dans l'espace qu'elle construit. C'est ce qu'on peut voir, respectivement, dans les énoncés suivants :

[...] avant tout, c'est Richard Feynman qui nous raconte ici la mécanique quantique. [Feynman R./Leighton R./Sands M. (1979, version française de B. Equer et P. Fleury). Le Cours de physique de Feynman, t. 3 («Mécanique quantique »). Paris : InterEditions, p. $\mathrm{x}$ ]

(6)

Comme les coefficients sont différents, nous écrivons $a_{n}=g e^{i k x n}+$ $d e^{-i k x n}$, pour $n \geq 1$. Ici $g$ est l'amplitude d'une onde allant vers la droite et $d$ celle d'une onde venant de la droite. [Le Cours de physique de Feynman, op. cit., p. 270] ${ }^{5}$

Dans l'exemple (5) (situé dans l'introduction de l'ouvrage), ici construit l'espace de l'énonciation comme étant celui du support matériel de l'écrit — un livre - espace englobant notamment celui qu'occupe l'occurrence de ici. Dans l'exemple (6), l'espace de l'énonciation rattaché à l'adverbe ici n'est pas le support matériel de l'écrit mais la linéarité écrite qui s'y inscrit sous la forme d'une ligne graphique. Aussi, là où il est possible de proposer la glose « dans ce livre » pour l'occurrence de ici de l'exemple (5), c'est, dans l'exemple (6), la glose « dans ces lignes » qui semble appropriée. Plus précisément encore, c'est la glose « dans la ligne qui précède » qui convient, l'occurrence de ici étant en effet alors exclue de l'espace qu'elle dessine. Dans les deux cas, ici désigne une étendue de texte non bornée et de taille plus ou moins importante, l'amplitude de cette étendue de texte étant toujours à interpréter en fonction de l'occurrence d'ici examinée. 


\section{Ci-dessous, plus haut, plus loin, « infra » : des SAdv métascripturaires}

Les SAdv ci-dessous, plus haut, plus loin et «infra» sont, comme l'adverbe ici étudié précédemment, susceptibles de soutenir des repérages déictiques spatiaux qui « rappellent» la nature écrite du dire. En ce sens, ils relèvent également du secteur «métascripturaire » de la sphère métadiscursive ${ }^{6}$. Le cadre du présent travail ne nous permettant pas de décrire précisément chacun de ces SAdv métascripturaires, nous ne livrerons ici que quelques remarques générales qui devraient être développées ultérieurement.

C'est tout d'abord par leur diversité formelle que ces SAdv se caractérisent. La locution adverbiale cidessous repose sur la composition de l'adverbe $c i$ (ici) avec l'adverbe dessous; plus haut et plus loin sont des groupes adverbiaux formés par l'association de l'adverbe de comparaison plus à, respectivement, un adjectif dans son emploi adverbial et un adverbe; enfin "infra » est une forme adverbiale latine traduisible par «au-dessous, plus bas ». On peut également noter que tous ces SAdv entrent dans la constitution d'une « paire » qui construit l'espace comme faisant l'objet d'une « bipartition » symétrique (ci-dessous/ci-dessus, plus haut/plus bas, «infra »/«supra ») ou non (plus loin/ici). Enfin, on remarque que ci-dessous et «infra»s'emploient exclusivement à l'écrit dans le cadre de la représentation d'une activité métascripturaire - autrement dit, leur usage est spécialisé —, alors que plus haut et plus loin peuvent être utilisés à l'écrit comme à l'oral, et indépendamment de toute activité métalangière?.

La prise en compte de l'ensemble de ces différences dans la description du fonctionnement référentiel de ces SAdv dépassant le cadre du présent travail, nous avons choisi de travailler sur la description de $c i$ dessous (/ci-dessus) en faisant l'hypothèse que les grandes caractéristiques que nous mettrons en évidence sont partagées par l'ensemble des SAdv métascripturaires. Considérons les SAdv ci-dessus et ci-dessous dans les exemples suivants :

(7)

Nous avons essentiellement considéré ci-dessus l'adaptation comme un phénomène génétique résultant de la sélection naturelle. En fait, il faut aussi considérer d'autres phénomènes qui peuvent conduire un individu d'un génotype donné à exprimer différents phénotypes. [Langaney A. (1988). Les hommes - passé, présent, conditionnel. Paris : Armand Colin, p.157]

(8)

Cette théorie de la signification trouve ses fondements dans la philosophie analytique. Nous en rappelons ci-dessous quelques présupposés de base sans la résumer de manière exhaustive. [Jeanneret T. (1999). La coénonciation en français - Approches discursive, conversationnelle et syntaxique. Bern, Berlin, Frankfurt/M., New York, Paris, Wien : Peter Lang, Éditions scientifiques européennes, p. 18-19]

En tant que composés de ici ( $c i$ dans sa forme abrégée), ci-dessus et ci-dessous partagent avec lui les propriétés que nous avons décrites précédemment : ils soutiennent un repérage spatial de type déictique et, au cours de cette opération, c'est un espace de nature écrite qui est construit comme espace de l'énonciation en cours. À la différence de ici cependant, ces SAdv permettent le repérage d'espaces distincts de celui de l'énonciation en train de se faire et n'ont qu'une interprétation, ils renvoient à la ligne écrite et, plus précisément, à une portion de la ligne écrite. Le lieu ainsi désigné est situé dans un « ailleurs » par rapport au lieu d'inscription du SAdv et, dans le cadre d'une représentation de l'espace en tant qu'orienté, est repéré comme en «amont » (dans le cas de ci-dessus) ou en « aval » (dans le cas de cidessous) relativement au lieu occupé par le SAdv.

En ceci, ces SAdv rappellent que le discours s'effectue dans un espace qui est celui de la ligne écrite, ligne graphique composée de signes linguistiques écrits qui, de ce fait, a des caractéristiques qui lui sont propres. Faire référence à un espace défini comme «en amont» ou « en aval », c'est ainsi souligner le caractère constitutif de la ligne écrite d'être ordonnée — en l'occurrence par la succession de gauche à droite et de haut en bas des signes $^{8}$ — cet ordre conférant une dimension chronologique à l'espace textuel, inscrivant le dire écrit dans ce qui apparait comme une « chronologie spatiale ». 


\section{Perspectives d'analyse}

Les caractéristiques référentielles des SAdv métascripturaires décrits en 2 et 3 permettent de dégager différents questionnements que nous évoquerons dans une visée programmatique, leur prise en compte étant en effet susceptible de contribuer, dans le cadre d'analyses de discours et de textes, à la différenciation de genres et d'écritures singulières. Si nous ne serons pas en mesure de mener des études de ce type dans la présente contribution, nous illustrerons notre propos par des analyses ponctuelles réalisées sur la base de deux corpus rassemblant différents écrits scientifiques relevant de champs disciplinaires distincts.

Le premier corpus (qui sera analysé en 4.1) est constitué en vue de l'étude des séquences métascripturaires comportant l'adverbe $i c i$; il se compose de quatre textes :

- un cours de mécanique quantique de premier cycle universitaire : Feynman R./Leighton R./Sands M. (1979, version française de B. Equer et P. Fleury). Le Cours de physique de Feynman, t. 3 (« Mécanique quantique »). Paris : InterEditions ;

- un essai de linguistique portant sur la grammaire de l'écrit : Gardes Tamine J. (2004). Pour une grammaire de l'écrit. Paris : Belin ;

- l'édition d'entretiens avec le philosophe René Girard : Girard R. (2004, traduction française/2008, éditions consultée). Les Origines de la culture. Entretiens avec Pierpaolo Antonello et Joao Cezar de Castro Rocha. Paris : Hachette ;

- $\quad$ et une lecture de l'œuvre de la psychanalyste Françoise Dolto : Brami E. et Delaroche P. (2014). Dolto, l'art d'être parents. L'éducation, la parole, les limites. Paris : Albin Michel.

Le second corpus (qui fera l'objet d'analyses en 4.2) est constitué en vue de l'étude des séquences métascripturaires comportant un SAdv du type de ci-dessus; il regroupe trois écrits de longueur variée qui peuvent être considérés, de différentes manières, comme des " synthèses »:

- une somme relative à l'histoire récente du Proche-Orient : Corm G. (2003). Le Proche-Orient éclaté. Paris : Gallimard;

- un essai philosophique se donnant pour tâche de caractériser la philosophie antique : Hadot P. (1995). Qu'est-ce que la philosophie antique?. Paris : Gallimard ;

- et une présentation, par un psychanalyste, des concepts majeurs développés par Jacques Lacan : Vanier A. (1998). Lacan. Paris : Les Belles Lettres.

Si nous serons ainsi amenée à proposer des interprétations qui nécessiteraient d'être vérifiées et étayées dans le cadre de corpus plus étendus ${ }^{9}$ que ceux que nous avons choisis, nous conserverons néanmoins ces interprétations, notre objectif étant ici d'abord de fournir des outils et de présenter une démarche pour des analyses à venir.

En guise de préambule, précisons qu'une première ligne de partage pourrait être tracée entre les genres et les écritures ne comportant pas de séquences métascripturaires, et les genres et les écritures qui, à l'inverse, en usent ${ }^{10}$. Dans les lignes qui suivent, il ne sera bien sûr question que de ce dernier cas de figure.

\subsection{Ici et ses interprétations : quels espaces pour l'énonciation écrite ?}

Considérant tout d'abord les deux interprétations possibles de $i c i$ - comme renvoyant au support matériel de l'écrit (le livre, par exemple), ou comme renvoyant à la ligne écrite - on pourrait se demander si l'espace de l'énonciation écrite, posé comme commun au scripteur et au lecteur, est donné comme celui de l'objet matériel qu'on tient entre ses mains, ou comme celui de la ligne écrite dont l'appréhension ne peut être que visuelle. Une première distinction pourrait ainsi être établie entre les 
genres ou entre les écritures selon qu'ils recourent à l'une seulement de ces deux interprétations ou aux deux.

De ce point de vue, il est possible dans notre corpus de rapprocher le cours de mécanique quantique de premier cycle universitaire de l'essai linguistique, ces deux ouvrages recourant en effet aux deux interprétations de $i c i$, comme nous l'avons déjà fait remarquer plus haut avec les exemples (5) et (6) pour le Cours de physique de Feynman (op. cit.), et comme on peut le voir dans les exemples suivants extraits de Pour une grammaire de l'écrit (op. cit.) :

La grammaire proposée ici est avant tout syntaxique (...). [Pour une grammaire de l'écrit, op. cit., p. 6]

Sauf précision contraire, c'est dorénavant dans ce seul sens syntaxique que le terme d'amplification sera utilisé ici. [Pour une grammaire de l'écrit, op. cit., p. 90]

Il ne s'agit pas ici de redire ce que d'autres ont exposé de façon détaillée. [Pour une grammaire de l'écrit, op. cit., p. 142]

Je me borne à signaler ici ce point qui sera repris plus loin (voir p. 172 à propos de la glose). [Pour une grammaire de l'écrit, op. cit., p. 158]

Le rôle des textes sera envisagé dans le chapitre suivant. Ne seront donc évoqués ici que quelques cas de fonctionnements locaux destinés à poser les problèmes. [Pour une grammaire de l'écrit, op. cit., p. 191]

Dans les exemples (9), (10) et (11), ici construit ainsi l'espace de l'énonciation en cours comme celui du livre, tandis que dans les exemples (12) et (13) l'espace de l'énonciation en cours correspond, respectivement, à la portion de la ligne écrite contenue dans la page sur laquelle s'inscrit l'occurrence de $i c i$, et à la portion de la ligne écrite qui constitue le chapitre auquel appartient l'occurrence de ici.

Notons que, dans ce premier groupe de textes où les deux interprétations de ici sont en présence, la prise en compte du mode selon lequel ces deux interprétations coexistent permettrait d'affiner les différenciations entre genres et entre écritures singulières. Il s'agirait alors de voir si ces deux interprétations alternent au fil de l'ouvrage - c'est le cas dans le Cours de physique de Feynman (op. cit.) et dans Pour une grammaire de l'écrit (op. cit.), le dire écrit y étant régulièrement rappelé dans son statut de « livre »-, ou bien si l'une de ces interprétations laisse définitivement sa place à l'autre dans le texte, cas dont notre corpus ne nous fournit pas d'exemple.

Dans un second cas de figure, les occurrences de ici réfèrent uniquement au support matériel de l'écrit, il en va ainsi dans l'édition d'entretiens avec le philosophe René Girard, et dans la lecture de l'œuvre de Françoise Dolto. Les occurrences de $i c i$ observées dans ces deux textes ne peuvent être glosées que par « dans ce livre ${ }^{11}$, comme on peut le voir dans les exemples suivants :

Mais si, dans ses livres précédents, René Girard avait surtout souligné les conséquences négatives d'un désir ainsi conçu, à savoir la dimension d'appropriation antagoniste, il évoque ici de façon plus explicite la valeur émancipatrice de l'imitation et (...). [Les Origines de la culture, op. cit., p. 10]

En réalité, comme cela apparaît clairement ici dans le chapitre «L'homme, un "animal symbolique" », l'apparition «fortuite » du sacré (...) [Les Origines de la culture, op. cit., p. 14]

L'approche par hypothèses et vérifications explicitement adoptée par Girard dans ses écrits a ici exigé une réflexion plus générale sur la méthodologie (...). [Les Origines de la culture, op. cit., p. 15] prolongements religieux de la théorie mimétique, le maillon faible de la «cathédrale 
girardienne », la dimension qu'il faudrait expulser d'une théorie qui sinon serait compatible avec le scepticisme ambiant. Girard montre ici que son discours est plus complexe. [Les Origines de la culture, op. cit., p. 17]

(18)

Afin de restituer le plus fidèlement et le plus simplement la pensée théorique et pratique de la célèbre psychanalyste, nous voulons ici donner à entendre ce que fut et demeure sa parole généreuse, sa démarche originale, susciter chez le lecteur la curiosité de retourner à ses textes. [Dolto, l'art d'être parents, op. cit., p. 10]

Dans chacun des cas de figure que nous venons d'évoquer, deux paramètres supplémentaires nous semblent particulièrement intéressants à prendre en compte. Il s'agirait d'interroger la façon dont les occurrences de ici sont réparties entre les différents «moments» de la structure interne du texte (avantpropos, introduction, développement, conclusion). Il conviendrait également d'être attentif à la distribution des occurrences de $i c i$ entre le texte et les éléments relevant de son «paratexte ${ }^{12}$ (par exemple, les notes de bas de page, la préface, la postface ou encore la quatrième de couverture).

Chacun des quatre textes examinés dans la présente section comporte au moins une occurrence de ces ici substituables par « dans ce livre » en introduction ou en avant-propos. Ces « seuils textuels » apparaissent ainsi, relativement aux autres «lieux» internes au texte, comme autant de zones de haute fréquence d'utilisation des occurrences de ici annonçant que l'élaboration du dire se fait dans un espace qui est le livre. On observe également une fréquence d'utilisation élevée ${ }^{13}$ de ce même emploi de ici dans les lieux paratextuels que sont la préface et la quatrième de couverture, cette dernière apparaissant comme l'un des premiers lieux de "rencontre ${ }^{14}$ du lecteur avec le texte produit par l'instance constituée du scripteur et de son éditeur, texte alors présenté d'emblée comme « fait livre », ainsi qu'on peut le voir dans les deux exemples relevés dans notre premier corpus ${ }^{15}$ :

Sous la forme d'entretiens avec deux universitaires qui l'interrogent sur son parcours, René Girard livre ici les principales étapes de son autobiographie intellectuelle. [Les Origines de la culture, op. cit., $4^{\mathrm{ème}}$ de couverture]

(20)

Ils [Elisabeth Brami et Patrick Delaroche] abordent ici les problèmes quotidiens en vue de soutenir dans leur tâche délicate les adultes trop souvent démunis. [Dolto, l'art d'être parents, op. cit., $4^{\text {ème }}$ de couverture]

L'étude des occurrences de $i c i$ dans les quatre textes que nous avons considérés dans cette section permet ainsi de poser l'hypothèse d'une affinité entre l'utilisation de ici comme renvoyant au support matériel de l'écrit et le discours tenu dans les moments et lieux inauguraux d'écrits académiques, que ces lieux soient de nature textuelle («Introduction », « Avant-propos », pour les textes étudiés), ou paratextuelle (ainsi de la «Préface» et de la quatrième de couverture). Le Cours de physique de Feynman (op. cit.) est exemplaire à ce titre, regroupant dans les 8 pages sur lesquelles s'étendent la préface et l'introduction de l'ouvrage, constitué de 517 pages, plus de la moitié des occurrences de ici interprétables comme «dans ce livre » relevées dans l'intégralité du volume, ceci avec une fréquence pouvant aller jusqu'à 2 occurrences par page, comme on pourra en juger en observant la liste de ces énoncés que reproduite ci-après dans son intégralité (et à laquelle on adjoindra l'exemple (5) cité plus haut) :

Les cours qui suivent ici ne sont pas considérés comme un survol, mais sont très sérieux. [Le Cours de physique de Feynman, op. cit., p. vi]

La méthode d'approche que vous trouverez ici est nouvelle, particulièrement pour le cours de deuxième année, et nous l'avons considérée surtout comme une expérience. [Le Cours de physique de Feynman, op. cit., p. ix]

Ce que vous trouverez ici, c'est le compte rendu de la première expérience. [Le Cours de physique de Feynman, op. cit., p. ix]

(...) pour la commodité de ceux qui ne disposent pas de ce volume, ces deux chapitres sont reproduits ici en appendice. [Le Cours de physique de Feynman, op. cit., p. $\mathrm{x}]$ 


\subsection{SAdv métascripturaires et dynamique de l'écrit}

Les occurrences des SAdv métascripturaires ci-dessous/ci-dessus, plus haut/plus bas, «infra »/《 supra » et plus loin - dorénavant «SAdv métascripturaires»- posent quant à elles la question de la localisation des portions de texte auxquelles elles font référence relativement à l'espace de l'énonciation en train de se faire : ces portions de texte se situent-elles en amont ou en aval relativement à l'espace occupé par le dire en train de se faire?

Nous voudrions montrer ici comment l'intérêt porté à ce qui apparaît comme la constitution d'une 《latéralisation » du texte permet de s'interroger sur les représentations de différentes « dynamiques » de l'écrit dont la prise en compte pourrait participer, là encore, à la différenciation de genres discursifs ou d'écritures singulières. Nos analyses se fondent sur les observations des séquences métascripturaires comportant un SAdv métascripturaire localisées dans les notes de bas de page des trois textes constituant le second corpus présenté plus haut. Dans ces trois textes, en effet, la séquence métascripturaire mettant en jeu un SAdv métascripturaire est, à de très rares exceptions près, voire toujours, exclue du corps du texte : seule une séquence métascripturaire comportant un SAdv métascripturaire a ainsi été relevée dans le corps du texte de l'ouvrage de G. Corm (1068 pages), de même dans l'ouvrage d'A. Vanier (118 pages); quant au corps du texte de l'ouvrage de P. Hadot (461 pages), il n'en contient aucune ${ }^{16}$. Étant donné le caractère massif de cette localisation en note des SAdv métascripturaires dans notre deuxième corpus, nous ne préciserons plus, à partir de maintenant, que les formes étudiées se situent en note.

Dans l'ouvrage de G. Corm, 14/27 SAdv métascripturaires (soit 51,8 \%) renvoient à une portion de texte relevant d'une antériorité spatiale relativement à l'espace de l'énonciation en cours, et 13/27 SAdv métascripturaires (soit 48,2\%) renvoient à une portion de texte située après l'espace de l'énonciation en cours, ainsi, respectivement, dans les exemples suivants :

Le Koweit fut un membre très actif de ces deux comités ${ }^{2}$.[[en note] 2. Voir ci-dessus, chapitre 12. [Le Proche-Orient éclaté, op. cit., p. 623]

Il voulut aussi islamiser la législation égyptienne, reniant l'œuvre laïque de ses prédécesseurs depuis Mohammmed Ali, en même temps qu'il prônait un retour à la démocratie libérale, aux libertés publiques et au pluralisme des partis ${ }^{2}$./[en note] 2. Sur le fondamentalisme religieux d'État, encouragé par les monarchies pétrolières, voir infra, chap. 7 . [Le Proche-Orient éclaté, op. cit., p. 264]

L'espace de l'énonciation en cours est ainsi posé de façon égale en relation à l'amont et à l'aval du dire.

Dans l'ouvrage de P. Hadot, 20/30 SAdv métascripturaires (soit 66,6 \%) font référence à une portion de texte localisée en amont relativement à l'espace de l'énonciation en cours, et 10/30 SAdv métascripturaires (soit 33,3\%) renvoient à une portion de texte située en aval comme, respectivement, dans les exemples suivants :

On remarquera à ce sujet qu'il semble bien que les présocratiques aient désigné leur démarche intellectuelle comme une historia, c'est-à-dire une enquête ${ }^{2}$./[en note] 2. Cf. plus haut, p. 29 ; (...). [Qu'est-ce que la philosophie antique ?, op. cit., p. 36]

Leur fondation correspond seulement à l'utilisation du droit d'association en vigueur à Athènes ${ }^{2}$./[en note] 2. Cf. plus bas, p. 156-157. [Qu'est-ce que la philosophie antique?, op. cit., p. 99]

Dans ce cas, l'espace de l'énonciation en cours est construit comme en relation privilégiée avec l'amont du dire.

Enfin, dans l'ouvrage d'A. Vanier, 4/11 SAdv métascripturaires (soit 36,4 \%) renvoient à une portion de texte localisée en amont relativement à l'espace de l'énonciation en cours, tandis que 7/11 SAdv métascripturaires (soit 63,6\%) désignent une portion de texte située en aval comme, respectivement, dans les exemples qui suivent : 
(29)

Il en retire des traits distinctifs - le trait unaire - qui lui permettent d'assumer cette image en se retournant vers le $\operatorname{miroir}^{16} . /[$ en note] 16 . Voir supra, chap. II. [Lacan, op. cit., p. 56]

(30)

Notre modernité montre bien l'urgence toujours renouvelée de cette tâche de civilisation qui est celle de la psychanalyse, ses enjeux ne se résumant pas à un « comment guérir?» - ce qui, pour autant, ne rend pas cette dimension subalterne comme en témoignent certaines des dernières interrogations de Lacan'./[en note] 2. Voir infra chap. V. [Lacan, op. cit., p. 13]

L'énonciation en cours apparait alors comme en relation privilégiée avec l'aval du dire.

Il est ainsi possible de dire que la dynamique de l'écrit représentée dans Qu'est-ce que la philosophie antique? est à " tendance rétrospective », tandis que celle qui est représentée dans Lacan est, en revanche, à «tendance prospective ». Dans Le Proche-Orient éclaté, la dynamique de l'écrit mise en scène dans les réalisations des différents SAdv métascripturaires peut être qualifiée d'«équilibrée 》 — à la fois rétrospective et prospective.

Les observations concernant la nature de cette dynamique de l'écrit représentée peuvent être affinées par l'étude du mode de coprésence des deux types de dynamique. De ce point de vue, on constate que, dans Qu'est-ce que la philosophie antique?, c'est chaque «lieu» du dire, qu'il soit situé au début, au milieu ou à la fin de la ligne écrite, qui est susceptible d'être mis en relation avec une antériorité ou avec une postériorité spatiale. En revanche, dans Le Proche-Orient éclaté et dans Lacan, la dynamique de l'écrit apparaît telle qu'organisée par rapport à ce que l'on peut appeler un «point de basculement ». Tandis que les SAdv métascripturaires renvoyant à une postériorité spatiale sont localisés dans ce qui est ainsi posé comme la partie inaugurale de la ligne écrite (dans leur intégralité, pour ce qui est de l'ouvrage d'A. Vanier, et dans leur grande majorité en ce qui concerne l'ouvrage de G. Corm), les SAdv métascripturaires renvoyant à une antériorité spatiale sont, quant à eux, situés dans la partie terminale de la ligne écrite (là aussi, dans leur intégralité, pour ce qui est de l'ouvrage d'A. Vanier, et dans leur grande majorité, en ce qui concerne l'ouvrage de G. Corm). Le "point de basculement", différemment fixé selon l'écrit envisagé, est donc le point de la ligne écrite à partir duquel se produit un changement dans la dynamique de l'écrit, qui, de prospective, devient rétrospective.

\subsection{Décrire l'activité métascripturaire}

Le type d'analyses que nous venons de présenter s'inscrit dans un ensemble plus vaste, relatif à la description de ce qu'on peut appeler l'«activité métascripturaire » représentée dans les séquences sur lesquelles nous nous penchons dans la présente contribution. L'adverbe $i c i$ et les SAdv métascripturaires que nous avons décrits apparaissent en effet comme des composants d'une activité réflexive relative au dire écrit qui engage d'autres ingrédients, dont l'examen permettrait de préciser la différenciation des genres discursifs et des écritures singulières du point de vue des séquences métascripturaires qu'on peut (ou pas) y observer. Nous énumérerons brièvement ces autres composants et les questions qu'ils soulèvent dans un dernier temps de manière à mettre en évidence l'arrière-plan sur lequel prennent place les éléments que nous avons évoqués.

Les syntagmes nominaux qui, très fréquemment (ainsi de «chap. V» dans l'exemple (30) ci-dessus) jouxtent les SAdv métascripturaires et dont le nom noyau est un nom référant à ce qu'on peut appeler une «part de texte ${ }^{17}$ (par exemple, « chapitre », «partie », mais aussi «page», ou encore «ligne») sont ainsi à analyser en ceci qu'ils construisent, eux aussi, des «lieux textuels» organisant l'espace dans lequel se déploie le dire écrit. Ainsi, dans «nous le verrons au chapitre 6 », ou encore dans «il en sera question dans le chapitre suivant», des unités textuelles définies dans le cadre d'une pratique de découpage du texte sont repérées, comme les SAdv métascripturaires que nous avons étudiés, relativement au lieu où le dire est en train de se faire.

L'examen des verbes qui constituent le centre (syntaxique) des séquences métascripturaires permet quant à lui de questionner la nature de l'activité «spatialisée ». Il s'agit alors de savoir quelles activités sont 
montrées comme prenant pour objet le dire écrit — «voir », « dire », « lire », « écrire », « revenir »?, pour ne citer que les verbes les plus fréquents.

Enfin, l'examen de la nature du (ou des) acteur(s) de cette activité permet de comparer les activités selon qu'elles sont attribuées au scripteur, au lecteur, ou au couple scripteur-lecteur, ceci selon différentes modalités dont nous donnons ci-dessous quelques exemples :

- mise en scène d'un « je » écrivant

(31)

Je reviendrai ci-dessous (2. à 5.) sur certains découpages opérables dans ce champ d'ensemble [...]. [J. Authier-Revuz J. (1995). Ces mots qui ne vont pas de soi. Boucles réflexives et non-coïncidences du dire, t.1. Paris : Larousse, p. 10]

- $\quad$ une écriture à « je » effacé

Il est clair que le champ, évoqué ci-dessus (2.2.1.), de la complexité énonciative telle qu'elle est saisie [...]. [Ces mots qui ne vont pas de soi, op. cit., p. 80]

- $\quad$ le « tu »-lecteur, un compagnon d'écriture

Nous retrouverons, plus loin, en (3.9.), les mécanismes d'identification et de construction d'images [...]. [J. Authier-Revuz, Ces mots qui ne vont pas de soi, op. cit., p. 346] ${ }^{1}$

- $\quad$ le lecteur indifférencié

Sur les différences d'approches entre les deux grandes tendances du sionisme, on verra le chapitre 7 qui traite en détail cette question. [Le Proche-Orient éclaté, op. cit., p. 647]

- le lecteur individualisé

Ceux qui ont préféré sauter le chapitre 6 devraient aussi sauter ce paragraphe. [Le Cours de physique de Feynman, op. cit., p. 251]

Le lecteur intéressé trouvera au chapitre 21 un complément d'information sur cette question, ainsi que sur la façon de désigner, en nomenclature, les deux configurations d'un carbone asymétrique. [Arnaud P. (1985). Cours de chimie organique. Paris : Éditions Gauthier-Villars, p. 40]

\section{Conclusion}

L'attention portée aux séquences métadiscursives contenant un syntagme adverbial faisant référence à un « lieu de l'écrit » sur le mode déictique peut fournir de nouvelles entrées pour l'analyse des textes et des discours. Mais elle contribue également à la réflexion menée dans deux autres domaines que nous évoquerons dans ce temps conclusif - le domaine intéressé à la cartographie de la sphère de l'activité métalangagière, et celui de la description de la « langue écrite».

Espaces d'une activité réflexive propre à l'énonciation en tant qu'elle se fait à l'écrit, c'est tout d'abord la " géographie » de la sphère du métadiscours (au sens de «métalangue en emploi et en action » (Authier et Doquet, 2012 : 18)) que ces séquences permettent de questionner. Dans Authier et Doquet (2012), les énoncés métadiscursifs sont ainsi distingués en fonction de leur objet, selon que celui-ci est A) le langage, la langue, ou B) le discours, et dans cette deuxième zone, a) le discours en train de se faire, posé en différence avec b) un discours autre ; ce que nous résumons comme suit en nous appuyant sur le schéma proposé dans Authier et Doquet (2012) dans lequel nous avons souligné les séquences métascripturaires introduites par nous :

\footnotetext{
${ }^{1}$ Dans cet exemple, la mise en gras de «3.9. » est de l'auteur.
} 


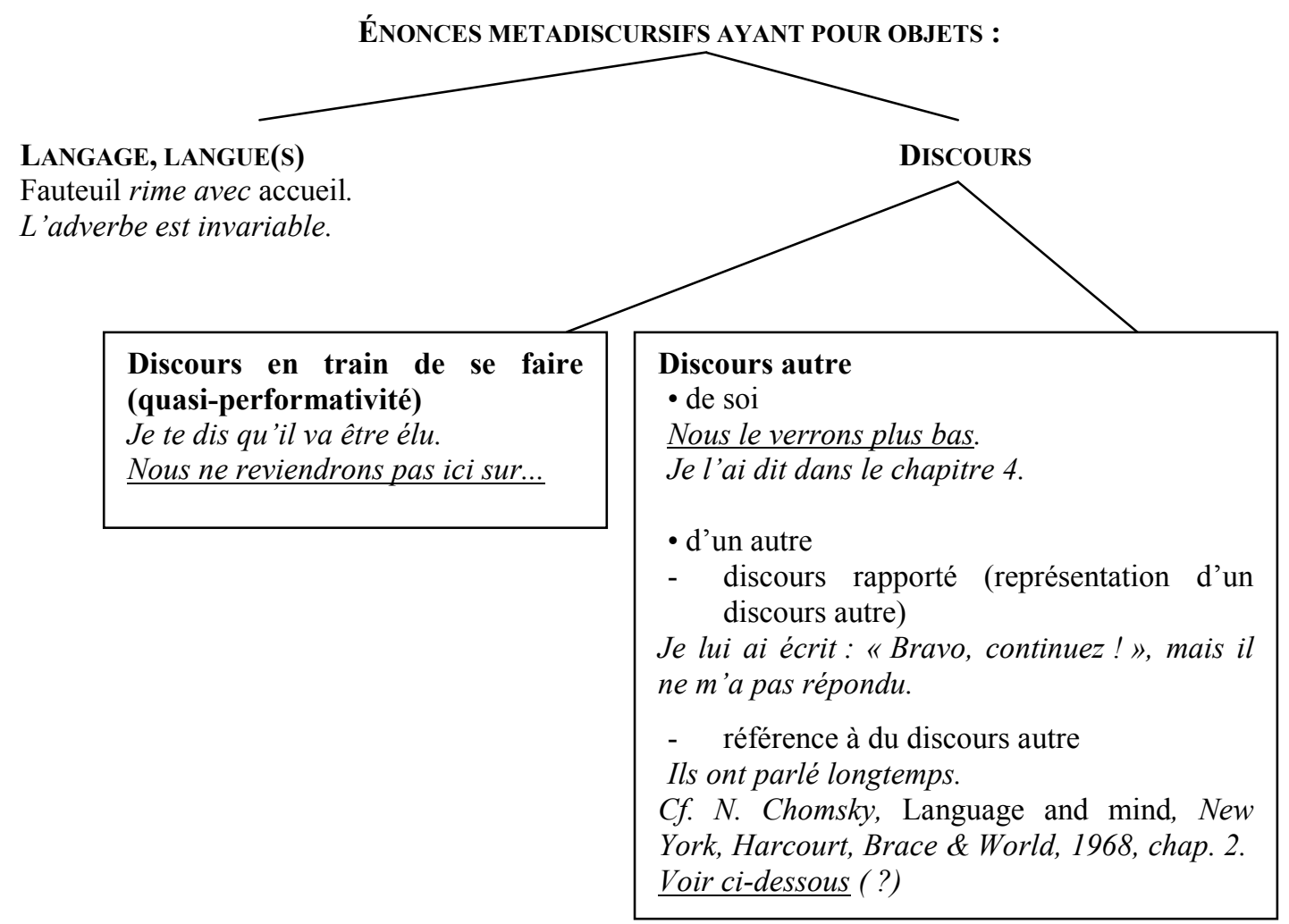

Eu égard à ces distinctions, les séquences du type de i) «nous ne reviendrons pas ici sur », du type de ii) " nous le verrons plus bas » et du type de iii) « voir ci-dessous », obligent à considérer l'existence d'un secteur transversal aux catégories proposées par Authier et Doquet (2012) et spécifiquement consacré à des formes i) de retour sur le discours en tant qu'il est en train de se faire à l'écrit; ii) de retour sur le discours autre de soi à l'écrit (en l'occurrence, dans l'exemple choisi, le discours qui sera tenu dans le futur de l'énonciation en cours); et iii) de retour sur le discours d'un autre à l'écrit, catégorie problématique en ce qu'elle suppose qu'on considère que dans une séquence telle que " voir ci-dessous ", l'activité de lecture équivaut au «discours» du lecteur. C'est ce secteur transversal que nous avons proposé de qualifier de « métascripturaire ».

Linéaire car constitué de signes linguistiques, le dire prend nécessairement place dans une chronologie. C'est ce que manifestent certaines séquences telles que «nous le verrons", " on l'a dit », ou encore " je reviendrai sur ce point plus tard ». Sans remettre en cause cette organisation du dire dans le temps, les séquences métascripturaires montrent que, à l'écrit, cette organisation se double d'une structuration du dire dans l'espace, structuration spécifique, à la différence de la première, à la langue et à l'énonciation écrites. Ainsi, parce que les SAdv qui occupent une place nodale dans ces séquences relèvent pour certains exclusivement de ce qui apparait comme la « langue écrite » (ainsi pour ci-dessus, par exemple), ou que la compréhension du mécanisme référentiel dont ils sont le lieu est indissociable de la prise en compte de la «substance» dans laquelle ils se réalisent (ainsi pour ici), c'est la nécessité d'une description de la langue écrite que ces énoncés métascripturaires mettent au premier plan, soulignant la pertinence toujours actuelle des propos de Nina Catach selon qui :

[1]oin d'effectuer un retour à une vision sclérosée du langage, qui confondrait la lettre et le son, et s'en tiendrait au texte, pris pour la langue, il nous faudra dorénavant, patiemment et posément, reconquérir la place de l'écrit auprès de l'oral, et ne pas avancer [...] sur un seul pied (Catach, $1988: 12){ }^{18}$ 


\section{Références bibliographiques}

Anis J. avec la collaboration de Chiss J.-L. et Puech C. (1988). L'écriture : théories et descriptions. Bruxelles : De Bœck-Wesmael.

Authier-Revuz J. (1995/2012). Ces mots qui ne vont pas de soi. Boucles réflexives et non-cö̈ncidences du dire. Limoges : Lambert-Lucas.

Authier-Revuz J. avec la collaboration de Doquet C. (2012). "Ce que je veux dire... - Accompagnements métadiscursifs d'une défaite de la parole. In Doquet C. et Richard E. (éds), Les représentations de l'oral chez Lagarce, Paris : Academia, 17-64.

Barbéris J.-M. (1994). Indiquer son chemin au passant: rôle cognitif et discursif de l'Orientation Générale. In Barbéris J.-M. (éd.), La ville - Arts de faire, manières de dire, Montpellier : Groupe de recherche en linguistique praxématique, collection Langue et praxis, Université Paul Valéry, 77-98.

Benveniste E. (1966). Problèmes de linguistique générale, t. 1. Paris : Gallimard.

Borillo A. (1985). Discours ou métadiscours. DRLAV, 32, 47-61.

Catach N. (éd) (1988). Pour une théorie de la langue écrite. Paris : Presses du C.N.R.S..

Laufer R. (1989). L'espace visuel du livre ancien. In Chartier R. et Martin H.-J. (éds), Histoire de l'édition française 1 - Le livre conquérant : du Moyen Age au milieu du XVII ème siècle. Paris : Fayard/Cercle de la librairie, 579-601.

Lefebvre J., à paraître. La note de bas de page. Étude sémiolinguistique et discursive. Limoges : Lambert-Lucas.

Perret M. (1982). De l'espace romanesque à la matérialité du livre. Poétique, 50, 173-182.

Perret M. (1988). Le signe et la mention - Adverbes embrayeurs ici, ça, la, iluec en moyen français (XIVè - XVè siècles). Genève : Librairie Droz.

Rey-Debove J. (1978/1997). Le métalangage. Paris : Armand Colin.

Simonin J. (1984). Les repérages énonciatifs dans les textes de presse. In Grésillon A. et Lebrave J.-L. (éd.), $L a$ langue au ras du texte, Lille : Presses Universitaires de Lille, 133-203.

Vachek J. (1939). Zum Problem der geschriebenen Sprache. Travaux du cercle linguistique de Prague, 8, 86-93 (traduction de P. Caussat, sous le titre «Contribution au problème de la langue écrite », Introduction par J.-L. Chiss et C. Puech in $\operatorname{LINX}, 12,7-23)$.

${ }^{1}$ Dans ces exemples ainsi que dans ceux qui suivront, le soulignement en gras est de nous.

${ }^{2}$ Les références des textes d'où sont issues les séquences métascripturaires que nous étudions sont indiquées entre crochets droits, en fin d'exemple.

${ }^{3}$ À l'écrit, l'adverbe ici est susceptible de permettre un repérage de type déictique - cas de figure qui nous intéresse actuellement —, ou de type anaphorique, comme dans l'exemple suivant (cité dans Simonin, 1984 : 195) : «Mais la crise a atteint Montpellier. Le chômage sévit ici, comme ailleurs ». Dans ce cas, la connaissance du contexte large de l'énoncé permet d'interpréter l'adverbe ici comme renvoyant à une "expression spatiale explicite du contexte» (ibid), en l'occurrence « Montpellier », et non au lieu géographique où le scripteur développe son dire.

${ }^{4}$ «C'est pourtant un fait à la fois original et fondamental que ces formes "pronominales" [soit je, tu, ici, maintenant] ne renvoient pas à la "réalité" ni à des positions "objectives" dans l'espace ou dans le temps, mais à l'énonciation, chaque fois unique, qui les contient, et réfléchissent ainsi leur propre emploi » (Benveniste, 1966 : 254).

${ }^{5}$ Dans la mise en texte d'origine que nous ne pouvons pas reproduire ici, «Comme les coefficients sont différents, nous écrivons » de même que « $a_{n}=g e^{i k x n}+d e^{-i k x n}$, pour $n \geq 1$. » sont suivis d'un passage à la ligne.

${ }^{6}$ Relativement à la dimension «méta » de ces SAdv, on notera que ci-dessus, ci-dessous, infra et supra font partie des «adverbes métalinguistiques» recensés dans l'étude du «métalexique » proposée dans Rey-Debove (1978/1997).

${ }^{7}$ Sur l'emploi non métascripturaire de plus haut et de plus loin, on pourra par exemple lire Barbéris 1994 qui étudie leur utilisation à l'oral dans la description d'itinéraires piétons dans un espace urbain. 


\footnotetext{
${ }^{8}$ Ces observations ne valent bien sûr que pour les écritures de type alphabétique ; il faudrait voir ce qu'il en est dans d'autres systèmes d'écritures, dans l'hypothèse où y existent également des séquences métascripturaires.

${ }^{9}$ Nous pensons par exemple au corpus «Scientext» développé à l’Université Grenoble 3 dans le but d' «étudier le positionnement et le raisonnement dans les écrits scientifiques ».

${ }^{10}$ À ce propos, il nous semble intéressant de remarquer ici que, contrairement à une idée communément partagée, les séquences métascripturaires ne sont pas exclues du champ littéraire, du moins, pour ce que nous avons pu constater pour le XXème siècle, de certains de ses secteurs. Ainsi peut-on lire par exemple dans Un captif amoureux: «Un peu plus haut, à propos de la déférence allant jusqu'à la flagornerie dans les mots, les inflexions, les gestes des feddayin devant les représentants palestiniens de la noblesse de banque ou d'Histoire, j'ai dit que je voulais revenir à Samia Solh » [Genet J. (1986). Un captif amoureux. Paris : Gallimard, p. 147].

${ }^{11}$ Lors de nos investigations, nous n'avons pas pu observer le cas dans lequel un texte aurait comporté uniquement des occurrences de ici paraphrasables par « dans ces lignes », aussi n'en donnons-nous pas d'exemples. On peut faire l'hypothèse que des textes à forte dimension exégétique ont exclusivement recours à ce type d'emploi de $i c i$.

${ }^{12}$ Voir Genette 1987.

${ }^{13}$ Relativement à la fréquence d'utilisation observée pour d'autres lieux paratextuels.

${ }^{14}$ Avec le titre figurant en première page de couverture.

${ }^{15}$ On remarquera que la présence en quatrième de couverture de l'adverbe ici comme équivalent de « dans ce livre » est également attestée dans notre second corpus, dans le texte d'A. Vanier où l'on peut lire : "On ne trouvera pas ici une explication du personnage Lacan mais une présentation de ses concepts majeurs (...) ».

${ }^{16}$ Sur la « distribution » des séquences métascripturaires entre le corps du texte et ses notes, voir Lefebvre à paraitre.

${ }^{17}$ Voir Lefebvre à paraître.

${ }^{18}$ Je tiens à remercier ici Michelle Lecolle et Jacqueline Authier-Revuz pour leurs lectures attentives et critiques.
} 